\title{
An Exploratory Analysis between the Feature Selection Algorithms $\mathrm{IG}_{\mathrm{MBD}}$ and $\mathrm{IG}_{\text {ChiMerge }}$
}

\author{
P.Kalpana \\ Department of Computer Science, Nehru Memorial College, Puthanampatti, 621 007, Tiruchirappalli (DT), India \\ E-mail: parasuramankalpana@gmail.com \\ K.Mani \\ Department of Computer Science, Nehru Memorial College, Puthanampatti, 621 007, Tiruchirappalli (DT), India \\ E-mail: nitishmanik@gmail.com
}

\begin{abstract}
Most of the data mining and machine learning algorithms will work better with discrete data rather than continuous. But the real time data need not be always discrete and thus it is necessary to discretize the continuous features. There are several discretization methods available in the literature. This paper compares the two methods Median Based Discretization and ChiMerge discretization. The discretized values obtained using both methods are used to find the feature relevance using Information Gain. Using the feature relevance, the original features are ranked by both methods and the top ranked attributes are selected as the more relevant ones. The selected attributes are then fed into the Naive Bayesian Classifier to determine the predictive accuracy. The experimental results clearly show that the performance of the Naive Bayesian Classifier has improved significantly for the features selected using Information Gain with Median Based Discretization than Information Gain with ChiMerge discretization.
\end{abstract}

Index Terms-ChiMerge Discretization, Feature Selection, Median Based Discretization, Naive Bayesian Classifier, Predictive Accuracy and Relevant Features.

\section{INTRODUCTION}

The ability of the Feature Selection (FS) process is to select the subset of prominent features and eliminate the features which provide little or no information for the prediction of class without distressing the performance of the classifier. It is also known as variable selection, variable subset selection, feature reduction or attribute selection. The benefits of feature selection are dimensionality reduction, better data visualization and understanding, storage reduction, speeding up the training and inference process and enhancing the model generalization. Because of these benefits now-a-days FS becomes an essential process in knowledge discovery. There are three major categories of models in subset selection. They are filters, wrappers and embedded model. Filters work independent of the predictor or classifier. The wrapper model uses the specific learning algorithm itself to assess the quality of the selected features. The embedded model performs variable selection in the process of training and is usually specific to given learning algorithms [18].

Discretization is a process which splits the range of continuous values into a small intervals and mapping each interval with a discrete symbol so as to provide useful information about the classes [12]. The discretization methods have been developed along different lines to meet different needs: supervised vs. unsupervised, splitting (top-down) vs. merging (bottomup), dynamic vs. static, global vs. local and direct vs. incremental. The supervised discretization method is more accurate because it uses the class information for discretization, whereas the unsupervised discretization does not use the class information but it is fast. The topdown discretization method starts with an empty list of cut-points and keeps on adding by splitting intervals whereas the bottom up method starts with the full list of all the continuous values of the feature as cut-points and gradually removes by merging the intervals. A dynamic discretization would discretize when the classifier is being built whereas the static discretization is carried out prior to the classification task. A local discretization would perform discretization in a localized region of instance space but a global method uses the entire instance space. Direct discretization divides the range of continuous attribute into $k$-interval simultaneously and the value of $k$ should be given by the user. The incremental method begins with simple discretization followed by an improvement process until stopping criteria is met [13].

The discretization process consists of four steps: i) sort the continuous values of a feature to be discretized ii) evaluate the cut-point for splitting or adjacent interval for merging iii) based on some criteria split or merge intervals of continuous values and iv) finally stop discretization [13].

The authors Mani.K and Kalpna.P (2015) have developed a novel FS method using Information Gain with Median Based Discretization $\left(I G_{M B D}\right)$ which is an unsupervised discretization method and proved that it provides better accuracy for Naive Bayesian Classifier $(N B C)$ for the selected features. It has also been proved that $I G_{M B D}$ selects more relevant features than IG with Equal Width Interval Discretization $\left(I G_{E W I D}\right)$, IG with 
Equal Frequency Interval Discretization $\left(I G_{E F I D}\right)$ and IG with Cluster Based Discretization $\left(I G_{C B D}\right)$ because the accuracy of the selected features using $I G_{M B D}$ is more than that of the others.

It is noted that the unsupervised discretization methods are usually faster but not normally efficient than supervised discretization (Jerzy W. Grzymala-Busse, 2013). Thus this paper performs a comparative analysis between $I G_{M B D}$, an unsupervised discretization method and Information Gain with ChiMerge discretization $\left(I G_{\text {ChiMerge }}\right)$, a supervised discretization method to determine which method provides better predictive accuracy for the $N B C$.

The rest of the paper is organized as follows. Section 2 depicts the related works. Section 3 describes the mathematical background required to understand the proposed methodology. The classification technique being used in this paper for prediction is presented in Section 4. Section 5 describes the comparison of $I G_{M B D}$ and $I G_{\text {ChiMerge }}$. The results are analyzed in section 5 . Finally section 6 ends with conclusion.

\section{RELATED WORKS}

An important issue related to mining large datasets both in dimension and size is of selecting a subset of original features using FS and this section provides an overview of several FS algorithms proposed in the literature.

Rajashree Dash et al, (2011) have discussed the basic concepts of discretization and different types of traditional supervised and unsupervised discretization techniques with examples. It was proved that an unsupervised method using k-means clustering gives equal performance as that of supervised methods viz., entropy based and chi-squared method [1]. James Dougherty et al, (1995) the authors compared the binning discretization methods with entropy-based and purity-based discretization methods. It was found that the performance of the Naive Bayes classifier is more using entropy-based discretization and proved that the discretized version of Naive Bayes algorithm slightly outperforms than C4.5. It was also observed that in some cases the performance of the C4.5 is significantly improved if the features are discretized in advance [3].

Ke wang et al. (1997) have proposed a minimum splits based discretization for continuous features to minimize the number of intervals with respect to impurity and proved that the proposed method generates a better decision tree [4]. The authors Salvador Garcia and et.al, (2013) have surveyed the discretization methods from the theoretical and empirical perspective. They tested the newest discretization methods using different types of classifiers for different datasets using different measures viz., accuracy, number of intervals and inconsistency [5]. Randy Kerber has described the ChiMerge, a general robust algorithm for discretization along with examples [6]. Arezoo Aghaei Chadegani and Davood Poursina (2013) have examined the of number of states for which the continuous variables are discretized will have the effect in the performance of Naive Bayes model and found that it provides best performance when the continuous variables are discretized into four states. It has also been identified that the performance deteriorates, when the number of states is increased to five or more [7].

The authors Derex D.Rucker et al, (2015) have showed that dichotomizing continuous variables via median based split and analyzing the resulting data via ANOVA involves a large number of costs and suggested to preserve the continuous nature of the variable and analyzing the data via linear regression [8]. A novel discretization method has been proposed by Daniela Joiţa (2010) based on k-means clustering algorithm which avoids the time required for the sorting the data items [10]. Jerzy W. Grzymala-Busse (2013) has launched entropy driven methodology for discretization with two enhancement options viz., dominant attribute and multiple scanning. They compared the proposed work with the well-known discretization methods viz., Equal Interval Width and Equal Frequency per Interval and proved that the entropy with multiple scanning is significantly better than the others [13].

Ying Yang and Geoffrey I. Webb (2002) have evaluated the effect of Naive Bayesian Classifier with nine discretization methods such as Equal Width Discretization (EWD), Equal Frequency Discretization (EFD), Entropy Minimization Discretization (EMD), Fuzzy Discretization (FD), Iterative Discretization (ID), Proportional K-interval Discretization (PKID), Lazy Discretization (LD), Non Disjoint Discretization (NDD) and Weighted Proportional K-interval Discretization (WPKID). It was found that the Naive Bayes Classifier with LD, NDD and WPKID achieves lower classification errors than of the others [14].

Nuntawut Kaoungku et al, (2013) have introduced a framework for missing value imputation and numerical data discretization using chi2 algorithm for classification and association rule mining and proved that the framework provides better results for both classification and association rule mining [15]. Prachya Pongaksorn et al. (2009) have designed a method for discretization called DCR, which uses both class information and the order between attributes to determine the discretization scheme with minimum number of intervals. They compared the DCR with five state of the art algorithms including two unsupervised and three supervised algorithms viz., EWID, EFID, CAIM, ChiMerge and WEKA discretization algorithm and proved that DCR requires fewer intervals, lower run time and provides high predictive accuracy than others [16].

K.Mani and P.Kalpana (2016) have developed a novel FS method using Bayes Theorem, Self Information and Sequential Forward Selection which uses the Median Based Discretization method for discretizing the continuous attributes into discrete and proved that their proposed work enhances the predictive accuracy for NBC [17].

From the existing literature it has been identified that many authors have introduced various novel methods for discretization and compared the same with the existing 
supervised and/or unsupervised discretization methods. In [2], the authors K.Mani and P.Kalpana (2015) have introduced a FS method called $I G_{M B D}$ and compared the same with three unsupervised discretization methods viz., $E W I D, E F I D$ and $C B D$ but they have not compared the same with any of the supervised discretization methods. Thus there is a need to compare the $I G_{M B D}$ with $\mathrm{IG}_{\text {ChiMerge }}$ and to verify the efficiency of the proposed work. Thus this paper performs the comparative analysis between $I G_{M B D}$ and $\mathrm{IG}_{\text {ChiMerge }}$

\section{MATHEMATICAL BACKGROUND}

This section describes the mathematical background required to understand the methodologies compared in this paper.

\section{A. Information Gain (IG)}

It is a univariate, entropy-based and symmetric FS method based on Claude Shannon on information theory and it determines the feature relevance between the attribute and class label in a supervised way (Lei Yu and Huan Liu, 2003).

The entropy of $X$ is computed as

$$
H(X)=-\sum_{i} P\left(x_{i}\right) \log _{2}\left(P\left(x_{i}\right)\right)
$$

The entropy of $X$ after observing $Y$ is computed as

$$
H(X \mid Y)=-\sum_{j} P\left(y_{j}\right) \sum_{i} P\left(x_{i} \mid y_{j}\right) \log _{2}\left(P\left(x_{i} \mid y_{j}\right)\right)
$$

Where $P\left(x_{i}\right)$ is the prior probability of all values of $X$ and $P\left(x_{i} \mid y_{i}\right)$ is the posterior probability of $X$ given $Y$. Thus the $I G$ for a feature $X$ and the class label $Y$ is calculated as

$$
I G(X \mid Y)=H(X)-H(X \mid Y)
$$

Where $H(X)$ is the entropy of $X$ and $H(X \mid Y)$ is the entropy of $Y$ after observing $X$.

\section{B. Pearson Chi-Square Test}

It is used to determine the significant between the attributes $A$ and $B$. Let $\left(A_{i}, B_{j}\right)$ denotes the joint event that attribute $A$ and $B$ takes on value $a_{i}$ and $b_{j}$ respectively. Then the $\chi^{2}$ is computed as

$$
\chi^{2}=\sum_{i=1}^{c} \sum_{j=1}^{r} \frac{\left(o_{i j}-e_{i j}\right)^{2}}{e_{i j}}
$$

Where $\mathrm{O}_{\mathrm{ij}}$ and $\mathrm{e}_{\mathrm{ij}}$ are the observed and expected frequencies of the joint event $\left(A_{i}, B_{j}\right)$ respectively and it is computed as

$$
e_{i j}=\frac{\operatorname{count}\left(A=a_{i}\right) \times \operatorname{count}\left(B=b_{j}\right)}{n}
$$

Where $n$ is the number of data tuples, count $\left(A=a_{i}\right)$ and count $\left(B=b_{j}\right)$ is the number of tuples having value $a_{i}$ for $A$ and $b_{\mathrm{j}}$ for $\mathrm{B}$ respectively. The $\chi^{2}$ statistic tests the null hypothesis $\left(\mathrm{H}_{0}\right)$ that $A$ and $B$ are independent with the significance level $\alpha=1 \%$ and $(r-1) \times(c-1)$ degrees of freedom, where $r$ and $c$ are the number of rows and columns in the contingency table respectively. If the computed $\chi^{2}$ value is greater than the tabulated value then $\mathrm{H}_{0}$ is accepted which means that $A$ and $B$ are correlated.

\section{ChiMerge Discretization}

It is a supervised discretization method based on $\chi^{2}$ test. It employs a bottom-up approach by finding the best neighboring intervals and then merging them to form larger intervals recursively. Initially, each distinct value of numeric attribute $A$ is considered to be one interval and $\chi^{2}$ tests are performed for every pair of adjacent intervals. Adjacent intervals with at least $\chi^{2}$ values are merged together, because low $\chi^{2}$ values for the pair indicates similar class distributions. This merging process continues recursively until a predefined stopping criterion is met.

\section{Computation of Median}

Median is a measure of central tendency, which exactly splits the data into two halves. There are several ways for computing median. This paper uses the median computation method used in discrete frequency distribution. i.e., it considers the item which has the value $\geq(N+1) / 2$, where $N=\sum f$ as median.

\section{Classification TechniQue}

There are three essential steps in the classification process. They are Model Construction (Learning), Model Evaluation (Testing) and Model Use (Classification). In the first phase, learning is performed on training data, which associates the class information and the classifier is built. In the second phase, the predictive accuracy of the model is computed based on test data. The percentage of test tuples that are classified correctly by the classifier from the test data becomes the accuracy of the classifier and the final step is used to classify the new instances [11].

\section{A. Naive Bayesian Classifier}

It is a statistical classifier based on the Bayes theorem. Let $D$ be a set of training tuples with class label. Suppose there are $m$ classes, $C_{1}, C_{2}, \ldots, C_{m}$. The role of this classifier is to predict that the given tuple $X$ belongs to the class having the highest posterior probability contained on $X$ (H. Liu et al, 2002). i.e., the tuple $X$ belongs to $C_{i}$ iff $P\left(C_{i} \mid X\right)>P\left(C_{j} \mid X\right)$ for $1 \leq j \leq m$ and $j \neq i . P\left(C_{i} \mid X\right)$ is computed as

$$
P\left(C_{i} \mid X\right)=\frac{P\left(X \mid C_{i}\right) P\left(C_{i}\right)}{P(X)}
$$


The accuracy of a classification method in general is the percentage of records that are classified correctly by the classifier in the test data and is computed as

$$
\text { Accuracy }=\frac{T P+T N}{T P+T N+F P+F N}
$$

Where True Positive (TP) refers to positive tuples that were correctly identified by the classifier, True Negative (TN) refers to negatives tuples that are correctly identified by the classifier, False Positive (FP) refers to negative tuples that are incorrectly classified and False Negative (FN) refers to positive tuples that were incorrectly labeled by the classifier (Jiawei Han, Jian Pei, and Micheline Kambar, 2011).

\section{COMPARISON OF MEDIAN BASED DisCRETIZED IG AND CHIMERGE DISCRTIZED IG}

The objective of the paper is to compare two FS methods viz., $I G_{M B D}$ and $I G_{\text {ChiMerge. }}$ The $M B D$ is an unsupervised, global, static and incremental method which uses splitting for discretization where as ChiMerge is a supervised, global, static and direct method which uses merging process for discretization. $I G$ is one of the popular measures used in $F S$. For continuous attributes the $I G$ considers the split which gives high $I G$ as the best split and it takes considerable among of time to find $I G$. The $I G_{M B D}$ uses median $(M)$ based discretized values for continuous features. I.e., it uses $M$ as the measure to split the continuous attributes into two halves and converts them into discrete based on $M$. It is an un-supervised discretization scheme and the corresponding pseudo code is shown below

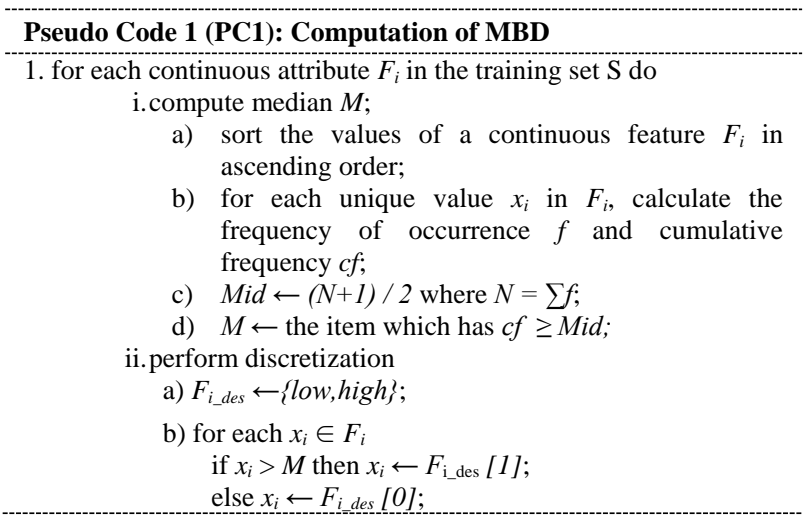

The main advantage of $M B D$ is that there is no need to specify the number of intervals because it splits the range of the continuous values into two halves and also there is no possibility for outliers. IG $G_{\text {ChiMerge }}$ uses ChiMerge discretized values for continuous features and its pseudo code is shown below.

\footnotetext{
Pseudo Code 2 (PC2): ChiMerge Discretization

1 . for each continuous attribute $F_{i}$ in the training set $S$ do

i. no_of_interval $\leftarrow$ number of distinct values in $F_{i}$

ii. place each distinct value of $F_{i}$ in one interval

iii. compute $\chi^{2}$ test for each adjacent intervals
}

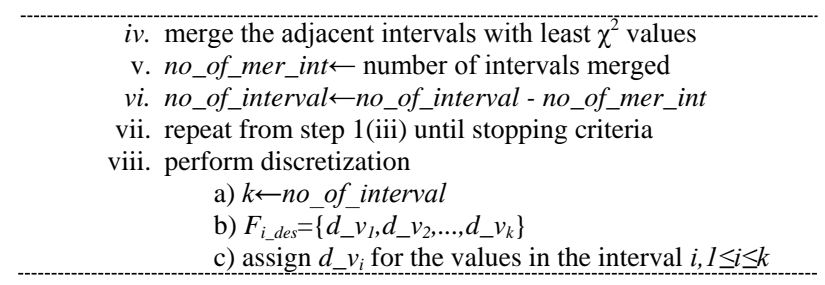

The discretized datasets obtained using both methods are then fed into $I G$, which computes the expected information for each attribute. The attributes are ranked based on their respective $I G$ 's. From the ranked attributes the attributes whose $I G$ is greater than the threshold are selected as the more relevant attributes where the threshold is fixed as $M$ of the $I G$ 's computed. The selected features of both methods are fed into the $N B C$ to determine the predictive accuracy.

\section{A. $I G_{M B D}:$ An Example}

In order to understand the relevance of the method $I G_{M B D}$, the weather dataset has been taken from the UCI machine learning repository and it is shown in Table 1.

Table 1. Weather dataset

\begin{tabular}{|c|c|c|c|c|}
\hline Outlook & Temperature & Humidity & Windy & Play \\
\hline Sunny & 85 & 85 & FALSE & No \\
\hline Sunny & 80 & 90 & TRUE & No \\
\hline Overcast & 83 & 86 & FALSE & Yes \\
\hline Rainy & 70 & 96 & FALSE & Yes \\
\hline Rainy & 68 & 80 & FALSE & Yes \\
\hline Rainy & 65 & 70 & TRUE & No \\
\hline Overcast & 64 & 65 & TRUE & Yes \\
\hline Sunny & 72 & 95 & FALSE & No \\
\hline Sunny & 69 & 70 & FALSE & Yes \\
\hline Rainy & 75 & 80 & FALSE & Yes \\
\hline Sunny & 75 & 70 & TRUE & Yes \\
\hline Overcast & 72 & 90 & TRUE & Yes \\
\hline Overcast & 81 & 75 & FALSE & Yes \\
\hline Rainy & 71 & 91 & TRUE & No \\
\hline
\end{tabular}

Table 2. Frequency and cumulative frequency for each unique values of temperature attribute

\begin{tabular}{|c|c|c|}
\hline $\begin{array}{c}\text { Unique values of } \\
\text { Temperature }\end{array}$ & $\begin{array}{c}\text { Frequency of } \\
\text { occurrence }(\boldsymbol{f})\end{array}$ & $\begin{array}{c}\text { Cumulative } \\
\text { frequency }(\boldsymbol{c} \boldsymbol{f})\end{array}$ \\
\hline 64 & 1 & 1 \\
\hline 65 & 1 & 2 \\
\hline 68 & 1 & 3 \\
\hline 69 & 1 & 4 \\
\hline 70 & 1 & 5 \\
\hline 71 & 1 & 6 \\
\hline $\mathbf{7 2}$ & $\mathbf{2}$ & $\mathbf{8}$ \\
\hline 75 & 1 & 10 \\
\hline 80 & 1 & 11 \\
\hline 81 & 1 & 12 \\
\hline 83 & 1 & 13 \\
\hline 85 & 1 & 14 \\
\hline
\end{tabular}

The dataset contains 5 fields viz., outlook, temperature, humidity, windy and play. Among these attributes, the 
temperature and humidity contain continuous values. As specified in PC1, the frequency $(f)$ and cumulative frequencies $(c f)$ of each sorted unique values $\left(x_{i}\right)$ of temperature attribute are computed and it is shown in Table 2.

From Table 2, Mid $=(14+1) / 2=7.5$ and hence $M=72$. Based on the $M$ the temperature is discretized as \{high, high, high, low, low, low, low, low, low, high, high, low, high, low . Similar procedure is applied to other continuous features too and the discretized values are shown in Table 3.

Table 3. Discretized values of continuous attributes in Weather dataset after $M B D$

\begin{tabular}{|c|l|}
\hline Temperature & $\begin{array}{l}\text { high, high, high, low, low, low, low, low, low, } \\
\text { high, high, low, high, low }\end{array}$ \\
\hline Humidity & $\begin{array}{l}\text { high, high, high, high, low, low, low, high, low, } \\
\text { low, low, high, low, high }\end{array}$ \\
\hline
\end{tabular}

After discretization the $I G$ is computed for all attributes. The entropy of play, $H$ (Play) is $-\left(9 / 14 \times \log _{2}\right.$ $\left.(9 / 14)+5 / 14 \times \log _{2}(5 / 14)\right)=0.94028$. The outlook feature contains 3 unique values viz., overcast, rainy and sunny. 4 instances of outlook contain overcast as its value. Out of these, all 4 instances have the value 'yes' for play attribute. Similarly 5 instances of outlook attribute contain the value 'rainy' and 'sunny' each. Out of the 5 rainy instances 3 contain 'yes' and 2 contain 'no' for play. Similarly out of the 5 sunny instances 2 contain 'yes' and 3 contain 'no' for play. Therefore the expected information needed to classify a tuple in weather dataset, if the tuples are partitioned according to outlook is

$H($ Outlook $\mid$ play $)=\left(4 / 14 \times\left(-\left(4 / 4 \times \log _{2}(4 / 4)\right)\right)\right)+(5 / 14 \times$ $\left.\left(-\left(3 / 5 \times \log _{2}(3 / 5)+2 / 5 \times \log _{2}(2 / 5)\right)\right)\right)+(5 / 14 \times(-(2 / 5 \times$ $\left.\left.\left.\log _{2}(2 / 5)+3 / 5 \times \log _{2}(3 / 5)\right)\right)\right)=0.69354$

Therefore, $I G($ Outlook $\mid$ Play $)=0.94028-0.64353$ $=0.24675$ using $(3)$.
Similar calculations are to be performed for other attributes too and the final ranking for the weather dataset is shown in Table 4. From the ranked attributes outlook and humidity are selected as the more relevant features because their $I G$ 's are greater than 0.0999 , where 0.0999 is the threshold, which is the median of the $I G$ 's in Table 4.

Table 4. Feature Ranking by IG with MBD

\begin{tabular}{|c|c|}
\hline Feature $\left(\mathbf{F}_{\mathbf{i}}\right)$ & IG(F $\left.\mathbf{F}_{\mathbf{i}}\right)$ after MBD \\
\hline Outlook & 0.2468 \\
\hline Humidity & 0.1518 \\
\hline Windy & 0.0481 \\
\hline Temperature & 0.0013 \\
\hline
\end{tabular}

B. $I G_{\text {Chimerge }}$ : An Example

Using chi-square test the critical values for the first two intervals of the feature temperature is shown in Table 5.

Table 5. Contingency Matrix

\begin{tabular}{|c|c|c|c|}
\hline \multirow{2}{*}{ Interval } & \multicolumn{2}{|c|}{ Play } & \multirow{2}{*}{ Total } \\
\cline { 2 - 4 } & Yes & No & \\
\hline I & $1(0.5)$ & $0(0.5)$ & 1 \\
\hline II & $0(0.5)$ & $1(0.5)$ & 1 \\
\hline Total & 1 & 1 & 2 \\
\hline
\end{tabular}

From table 5, o $11=1, o_{12}=0, o_{21}=0$ and $o_{22}=1, e_{11}=$ $(1 \times 1) / 2=0.5, e_{12}=(1 \times 1) / 2=0.5, e_{21}=(1 \times 1) / 2=0.5$ and $e_{22}=(1 \times 1) / 2=0.5$ and the $\chi^{2}$ statistics is calculated as

$\chi^{2}=(1-0.5)^{2} / 0.5+(0-0.5)^{2} / 0.5+(0-0.5)^{2} / 0.5+(1-0.5)$ $/ 0.5=2$.

The table $\chi_{0.01,1}^{2}=6.635$. Since the calculated $\chi^{2}(2)<$ the table value (6.635), the intervals are merged. Similar computation is performed for other adjacent pair of intervals and they are shown in Table 6.

Table 6. Demonstration of ChiMerge Discretization

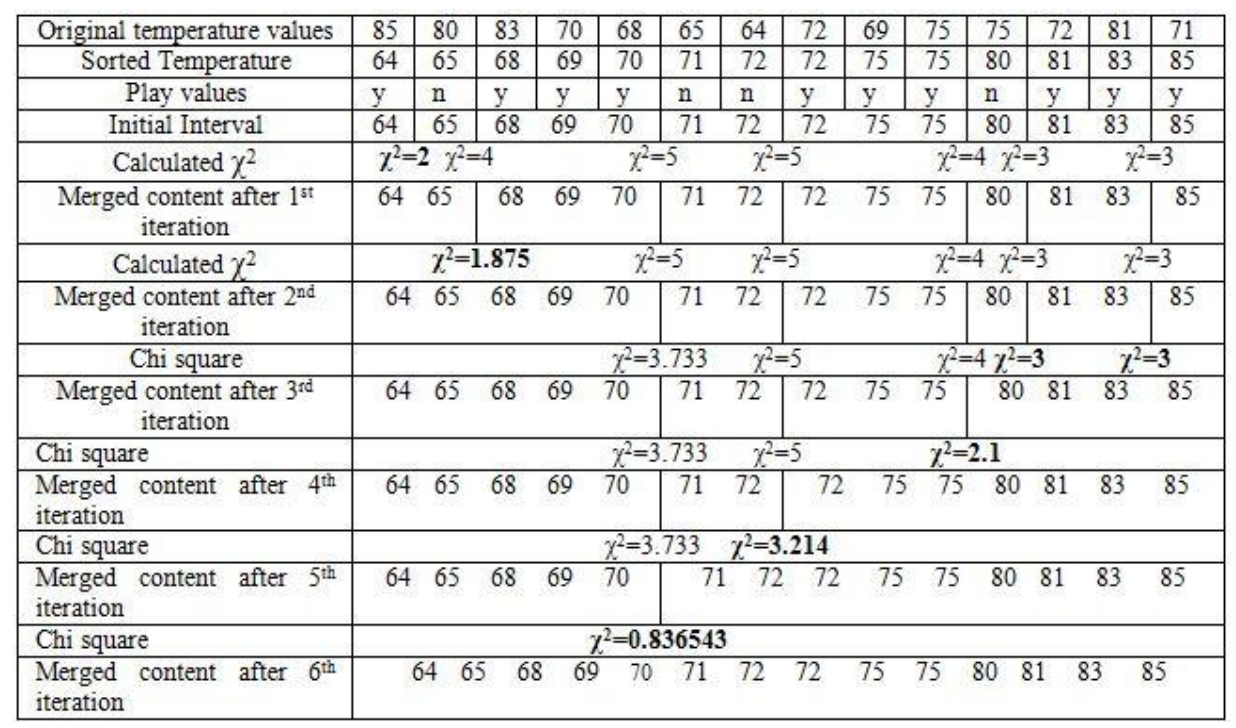


After finding the critical values, the first two intervals are merged together because it has least $\chi^{2}$ value. This process is repeated until the calculated $\chi^{2}$ value is greater than 6.635 or when all items of a feature fall in the same interval. From table 5, it is observed that after 6 iterations, all values of temperature fall in one interval and it is discretized as [true, true, true, true, true, true, true, true, true, true, true, true, true, true]. Similar procedure is applied to other continuous attributes too and the discretized values of the continuous attributes temperature and humidity of weather dataset after ChiMerge discretization is shown in Table 7.

Table 7. Discretized values of continuous attributes in Weather dataset after ChiMerge discretization

\begin{tabular}{|l|l|}
\hline Temperature & $\begin{array}{l}\text { high, high, high, high, high, high, high, high, } \\
\text { high, high, high, high, high, high }\end{array}$ \\
\hline Humidity & $\begin{array}{l}\text { high, high, high, high, high, high, high, high, } \\
\text { high, high, high, high, high, high }\end{array}$ \\
\hline
\end{tabular}

After discretization, the $I G$ is computed for the discretized dataset as in section 5(A) and the ranking are shown in Table 8.

Table 8 . Feature Ranking by $\mathrm{IG}_{\text {ChiMerge }}$

\begin{tabular}{|c|c|}
\hline Feature $\left(\mathbf{F}_{\mathbf{i}}\right)$ & IG( $\left.\mathbf{F}_{\mathbf{i}}\right)$ after ChiMerge \\
\hline Outlook & 0.2468 \\
\hline Windy & 0.0481 \\
\hline Humidity & 0.0000 \\
\hline Temperature & 0.0000 \\
\hline
\end{tabular}

The attributes outlook and windy are selected from the ranked attributes as the more relevant feature because their $I G$ 's are greater than 0.0241 .

\section{EXPERIMENTAL STUDY}

In order to analyze the said methods, 6 datasets have been taken from UCI Machine Learning Repository [9]. Each dataset contains both continuous and nominal features. The missing values for each attribute in the datasets are filled with their corresponding mean. The detailed specification of these datasets is shown in Table 9.

Table 9. Description of the datasets

\begin{tabular}{|c|c|c|c|}
\hline Dataset & $\begin{array}{c}\text { No. of } \\
\text { Attributes }\end{array}$ & $\begin{array}{c}\text { No. of } \\
\text { Instances }\end{array}$ & $\begin{array}{c}\text { No. of } \\
\text { Classes }\end{array}$ \\
\hline $\begin{array}{c}\text { Pima Indian } \\
\text { Diabetes }\end{array}$ & 9 & 768 & 2 \\
\hline Breast Cancer & 11 & 699 & 2 \\
\hline Statlog Heart & 14 & 270 & 2 \\
\hline Eeg & 15 & 14979 & 2 \\
\hline Weather & 5 & 14 & 2 \\
\hline Ann-train & 22 & 3772 & 3 \\
\hline
\end{tabular}

The $I G_{M B D}$ has been implemented in Python, ChiMerge discretization is obtained using chiM function of 'discretization' package in $\mathrm{R}$ tool and $I G$ is using WEKA tool. The original features and the selected features using
$I G_{M B D}$ and $I G_{\text {ChiMerge }}$ discretization are fed into the $N B C$ and accuracies are computed using WEKA tool with 10fold cross validation technique. The results are shown in Table 10.

Table 10. Comparison of Predictive Accuracy of NBC for the selected features using $\mathrm{IG}_{\text {ChiMerge }}$ and $\mathrm{IG}_{\mathrm{MBD}}$

\begin{tabular}{|c|c|c|c|c|}
\hline \multirow{3}{*}{ Dataset } & \multirow{3}{*}{$\begin{array}{c}\text { \#Selected } \\
\text { features }\end{array}$} & \multicolumn{3}{|c|}{ Accuracy (\%) of NBC with } \\
\hline & & \multirow{2}{*}{$\begin{array}{c}\text { All } \\
\text { Features }\end{array}$} & \multicolumn{2}{|c|}{$\begin{array}{c}\text { Selected Features } \\
\text { using }\end{array}$} \\
\hline & & & IG $G_{\text {ChiMerge }}$ & $I G_{M B D}$ \\
\hline $\begin{array}{c}\text { Pima } \\
\text { Indian } \\
\text { Diabetes }\end{array}$ & 4 & 76.3021 & 75.3906 & 76.5625 \\
\hline $\begin{array}{l}\text { Breast } \\
\text { cancer }\end{array}$ & 5 & 95.9943 & 95.9943 & 95.2790 \\
\hline $\begin{array}{l}\text { Statlog } \\
\text { heart }\end{array}$ & 6 & 83.7037 & 85.1852 & 85.1852 \\
\hline Eeg & 7 & 48.0406 & 52.2131 & 48.9285 \\
\hline Weather & 2 & 64.2857 & 57.1429 & 71.4286 \\
\hline Ann-train & 10 & 95.6522 & 95.5992 & 95.7317 \\
\hline \multicolumn{2}{|c|}{ Average } & 77.3298 & 76.9209 & 78.8526 \\
\hline
\end{tabular}

The number of original features and the selected features of both methods are shown in Figure 1. Since median $I G$ 's is fixed as threshold, both methods select the same number of attributes approximately $50 \%$. Figure 2 shows the accuracy comparison of $N B C$ with all features, selected features with $I G_{M B D}$ and $I G_{\text {ChiMerge }}$.

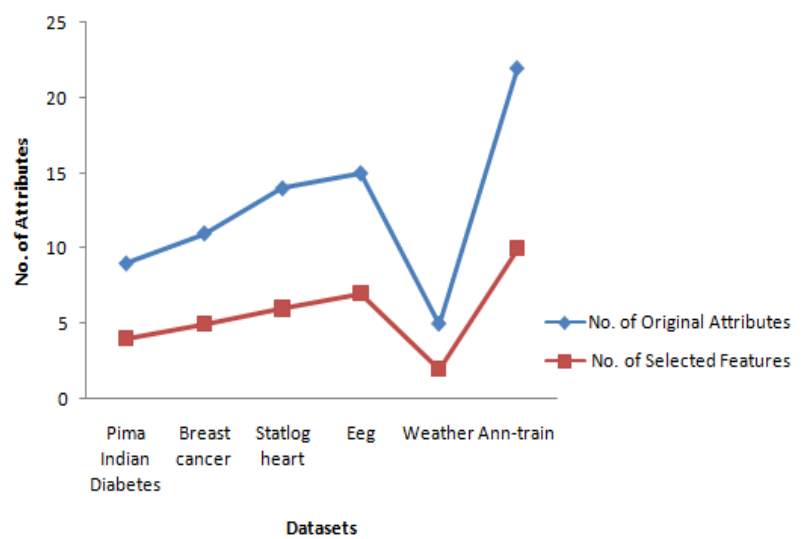

Fig.1. Comparison of number of original features selected features using $\mathrm{IG}_{\mathrm{MBD}}$ and $\mathrm{IG}_{\text {ChiMerge }}$

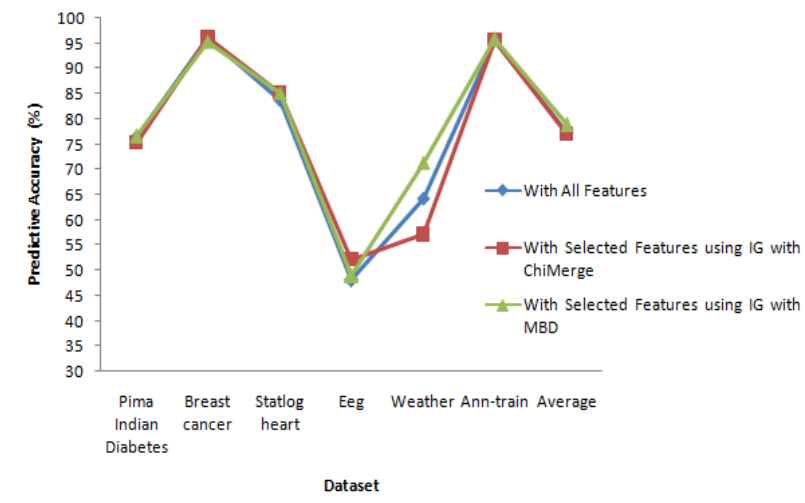

Fig.2. Comparison of predictive accuracy of NBC with all features and with selected features using $\mathrm{IG}_{\mathrm{MBD}}$ and $\mathrm{IG}_{\text {ChiMerge }}$ 
From table 10, it is observed that, the accuracy of Pima Indian Diabetes, Weather and Ann-train is greater using the selected features with $I G_{M B D}$. Similarly the accuracy of Breast cancer and Eeg, the accuracy of $N B C$ has greater value for the selected features with $I G_{\text {ChiMerge }}$. The accuracy of Statlog heart remains the same for both methods. It is observed that the accuracy of the selected features using $I G_{M B D}$ is $78.8526 \%$ on an average which is greater than the selected features using $I G_{\text {ChiMerge }}$ $(76.9209 \%)$ and also with original features (77.3298\%).

This is because $M B D$ uses median which is one of the measure of central tendency and it splits the continuous attributes into two halves which ultimately leads no two values fall in the same group where as the ChiMerge discretizes uses ' $n$ ' iterations, each comprising of chisquare test and merging intervals. It is noted that a minimum number of discrete intervals reduce the data size which results in better understanding of the discretized attributes. $I G_{M B D}$ has two intervals where as $I G_{\text {ChiMerge }}$ has ' $i$ ' intervals where $1 \leq i \leq n$ and ' $n$ ' is the maximum number of intervals. As $I G_{M B D}$ has only two intervals and provides more accuracy than $I G_{\text {ChiMerge, }}$ it is proved that $I G_{M B D}$ is better than $I G_{\text {ChiMerge. }}$.

\section{CONCLUSION}

This paper compares $I G_{M B D}$ with $I G_{\text {ChiMerge }}$ by measuring the accuracy of $N B C$ on several different datasets. It is evident from the table that the accuracy of the $N B C$ using $I G_{M B D}$ is increased by $1.9317 \%$ on an average when compared with $I G_{\text {ChiMerge. }}$ It is also proved that for the reduced feature set, there is an accuracy enhancement of $1.5228 \%$ than the original features. The result clearly shows that the model constructed using $I G_{M B D}$ performs well for $N B C$ and is equally competent with $\mathrm{IG}_{\text {ChiMerge }}$. Further both methods reduce the features by $50 \%$ which reduces the classification time and the space requirements too.

\section{REFERENCES}

[1] Rajashree Dash, Rajib Lochan Paramguru and Rasmita Dash, "Comparative Analysis of Supervised and Unsupervised Discretization Techniques", International Journal of Advances in Science and Technology, vol. 2, no. 3, pp. 29-37, 2011.

[2] K. Mani and P. Kalpana, "A Filter-based Feature Selection using Information Gain with Median Based Discretization for Naive Bayesian Classifier", International Journal of Applied and Engineering Research, vol. 10, no.82, pp. 280-285, 2015.

[3] James Dougherty, Ron Kohavi and Mehran Sahami, "Supervised and Unsupervised Discretization of Continuous Features (Published Conference Proceedings style)", In Proceedings of the $12^{\text {th }}$ International Conference, Morgan Kaugmann Publishers, vol. 25, pp.194-202, 1995.
[4] Ke Wang and Han Chong Goh, "Minimum Splits Based Discretization for Continuous Features", IJCAI, vol. 2, pp. 942-951, 1997.

[5] Salvador Garcia, Julian Luengo, Jose Antonio Saez, Victoria Lopez and Francisco Herrera, "A Survey of Discretization Techniques: Taxonomy and Empirical Analysis in Supervised Learning", IEEE transactions on Knowledge and Data Engineering, vol. 25, no. 4, pp.734750, 2013.

[6] Randy Kerber, "ChiMerge: Discretization of Numeric Attributes (Published Conference Proceedings style)", In Proceedings of the tenth national conference on Artificial Intelliegence, Aaai press, pp. 123-128, 1992.

[7] Arezoo Aghaei Chadegani and Davood Poursina, "An examination of the effect of discretization on a naïve Bayes model's performance", Scientific Research and Essays, vol. 8, no. 44, pp. 2181-2186, 2013.

[8] Derex D.Rucker, Blakeley B.McShane and Kristopher J.Preacher, "A Research 's guide to regression, discretization and median splits of continuous variables", Journal of Consumer Psychology, Elsevier, vol. 25, no. 4, pp. 666-668, 2015.

[9] UCI Machine Learning Repository - Center for Machine Learning and Intelligent System, Available: http://archive.ics.uci.edu.

[10] Daniela Joiţa, "Unsupervised Static Discretization Methods in Data Mining", Titu Maiorescu University, Bucharest, Romania, 2010.

[11] Jiawei Han, Jian Pei, and Micheline Kambar, "Data Mining: Concepts and Techniques", $3^{\text {rd }}$ edition, Elsevier, 2011.

[12] H.Liu, F.Hussain, C.L.Tan, and M.Dash, "Discretization: An Enabling Technique", Data Mining and Knowledge Discovery, vol. 6, no. 4, pp. 393-423, 2002.

[13] Jerzy W. Grzymala-Busse, "Discretization Based on Entropy and Multiple Scanning", Entropy, vol. 15, no. 5, pp.1486-1502, 2013.

[14] Ying Yang and Geoffrey I. Webb, "A Comparative Study of Discretization Methods for Naive-Bayes Classifiers (Published Conference Proceedings style)", In Proceedings of PKAW, vol. 2002, pp. 159-173, 2002.

[15] Nuntawut Kaoungku, Phatcharawan Chinthaisong, Kittisak Kerdprasop, and Nittaya Kerdprasop, "Discretization and Imputation Techniques for Quantitative Data Mining (Published Conference Proceedings style)", In Proceedings of International MultiConference of Engineers and Computer Scientists, MECS, vol. 1, 2013.

[16] Prachya Pongaksorn, Thanawin Rakthanmanon, and Kitsana Waiyamai "DCR: Discretization using Class Information to Reduce Number of Intervals", Quality issues, measures of interestingness and evaluation of data mining models (QIMIE'09), pp.17-28, 2009.

[17] K.Mani, P.Kalpana, "An Efficient Feature Selection based on Bayes Theorem, Self Information and Sequential Forward Selection", International Journal of Information Engineering and Electronic Business(IJIEEB), vol.8, no.6, pp.46-54, 2016. DOI: 10.5815/ijieeb.2016.06.06.

[18] Saptarsi Goswami, Amlan Chakrabarti, "Feature Selection: A Practitioner View", International Journal of Information Technology and Computer Science, vol.11, pp.66-77, 2014. DOI: 10.5815/ijitcs.2014.11.10 


\section{Authors' Profiles}

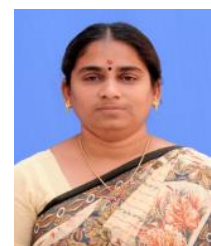

P. Kalpana received B.Sc and M.Sc degrees in Computer Science from Seethalakshmi Ramaswami College, affiliated to Bharathidasan University, Tiruchirappalli, India in 1999 and 2001 respectively. She received M.Phil degree in Computer Science in 2004 from Bharathidasan University. She also received MBA degree in Human Resource Management from Bharathidasan University in 2007. She is presently working as an Assistant Professor in the Department of Computer Science, Nehru Memorial College, Puthanampatti, Tiruchirappalli. She is pursuing $\mathrm{PhD}$ degree in Computer Science at Bharathidasan University. Her research interests include Algorithms, Data Pre-processing and Data Mining techniques.

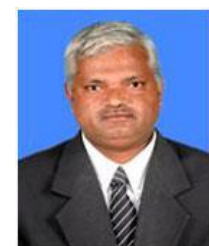

K. Mani received his MCA and M.Tech. from the Bharathidasan University, Trichy , India in Computer Applications and Advanced Information Technology respectively. Since 1989 , he has been with the Department of Computer Science at the Nehru Memorial College, affiliated to Bharathidasan University where he is currently working as an Associate Professor. He completed his $\mathrm{PhD}$ in Cryptography with primary emphasis on evolution of framework for enhancing the security and optimizing the run time in cryptographic algorithms. He published and presented around 15 research papers at international journals and conferences.

How to cite this paper: P.Kalpana, K.Mani, "An Exploratory Analysis between the Feature Selection Algorithms IG $_{\mathrm{MBD}}$ and IG $_{\text {ChiMerge" }}$ ", International Journal of Information Technology and Computer Science(IJITCS), Vol.9, No.7, pp.61-68, 2017. DOI: 10.5815/ijitcs.2017.07.07 